Corresponding Author:

J. Viñán

janneth.vinan@espoch.edu.ec

Received: 10 January 2020

Accepted: 17 January 2020

Published: 26 January 2020

Publishing services provided by Knowledge E

(c) J. Viñán et al. This article is distributed under the terms of the Creative Commons

Attribution License, which permits unrestricted use and redistribution provided that the original author and source are credited.

Selection and Peer-review under the responsibility of the $\mathrm{VI}$ Congreso Internacional Sectei 2019 Conference Committee.

\section{Impacto Financiero Y La Rotación De Personal En Las Áreas De Mercadeo Y Operaciones: Empresa Multinacional De Venta De Vacaciones Prepagadas En El Ecuador}

\section{Financial Impact and the Rotation of Personnel in the Marketing Areas and Operations: Multinational Company of Sale of Prepaid Vacations in Ecuador}

\author{
J. Viñán ${ }^{1}$, G.Miño ${ }^{2}$, J. Arias ${ }^{1}$, and A. Viterí ${ }^{3}$ \\ ${ }^{1} \mathrm{ESPOCH}$ Facultad de Administración de Empresas \\ ${ }^{2}$ ESPOCH Facultad Mecánica \\ ${ }^{3}$ Decamerón, Talento Humano Duque
}

\section{Resumen}

El talento humano es determinado como el aporte más valioso en toda empresa, principalmente en las empresas de servicio, por lo que uno de los principales retos es potenciar al talento, buscando estrategias que identifiquen las necesidades y motivaciones que permita brindar agradado, desarrollo profesional y personal a los colaboradores. La ineficaz motivación y el poco compromiso que posee el personal genera un impacto en la rotación de personal lo que se verá reflejado a nivel financiero de la compañía; se ha identificado que la continua y excesiva rotación de personal es perjudicial para el cumplimiento de los objetivos empresariales, así como los costos que se genera en los procesos de reclutamiento, selección, inducción, capacitación, entre otros; y también al momento de la desvinculación. El principal objetivo del estudio es determinar el impacto financiero de la rotación de personal de las áreas de mercadeo y operaciones en una Empresa Multinacional de Venta de Vacaciones Prepagadas en el Ecuador. Ya que la incertidumbre, inestabilidad, genera alta rotación en especial a los nuevos postulantes, y los antiguos trabajadores que réquiem de estabilidad para el cumpliendo de sus objetivos profesionales. En la rotación de personal la organización debe incurrir en un conjunto de desembolso diarios obligatorios por Ley en la desvinculación del personal saliente demás de los requeridos para el reclutamiento y contratación de quienes cubrirán las vacantes presentadas.

Abstract: Human talent is considered the most valuable contribution in every company, mainly in service companies, so one of the challenges is to maximize the talent looking for strategies to identify needs and motivations that allow providing gratified, professional development, personal. Ineffective motivation and commitment that has 
little staff has an impact on the turnover which will be reflected in financial terms, has been identified as the continued and excessive staff turnover is detrimental to the fulfillment of business objectives, as the costs generated in the process of recruitment, selection, induction, training, among others; and also at the time of termination. The main objective of the study is to determine the financial impact of staff turnover in the areas of marketing and operations in a multinational company selling Prepaid Vacations in Ecuador. As uncertainty, instability, high turnover generated in particular to new applicants, and former employees who requiem stability for fulfilling their career goals. Turnover in the organization must incur a set of mandatory daily payout by law in the decoupling of outgoing personnel other than those required for the recruitment and hiring of those who fill the vacancies presented.

Palabras Clave: Talento humano, rotación de personal, costos, reclutamiento y contratación.

Keywords: human talent, turnover, recruiting and hiring costs.

\section{Introducción}

La gestión del talento humano se orienta a la satisfacción, empoderamiento de los colaboradores desde el manejo de las herramientas de trabajo más usuales hasta la conformación de grupos de trabajo de alto rendimiento; de esta manera se harán personas competitivas en el mundo globalizado. El talento humano es considerado el aporte más valioso en toda empresa, principalmente en las empresas de servicio, por lo cual uno de los retos es potenciar al máximo al talento buscando estrategias que permitan identificar las necesidades y motivaciones que permita brindarles agradado, desarrollo profesional, personal. La ineficaz motivación y el poco compromiso que posee el personal genera un impacto en la rotación de personal lo que se verá reflejado a nivel financiero, se ha identificado que la rotación continua y excesiva de personal es perjudicial para el cumplimiento de los objetivos empresariales, así como los costos que se genera en los procesos de reclutamiento, selección, inducción, capacitación, entre otros; y también al momento de la desvinculación.

La rotación de personal es un problema que se ha convertido en una constante, debido a que ha presentado altos porcentajes de desvinculación de personal, lo que ha generado inestabilidad laboral en los colaboradores, y problemas para cubrir puestos vacantes en corto plazo, posiblemente debido a la baja remuneración y la necesidad 
de cumplir metas previamente establecidas; lo que ha provocado reclutamientos incorrectos, capacitaciones inconclusas, y adaptación en la adecuación al puesto.

Después de un análisis a los indicadores que se emplean mensualmente por el área de Recursos Humanos, se ha determinado que existe un alto índice de rotación en las áreas de Mercadeo y Operaciones.

Por lo que como parte de las estrategias de Gestión del Talento Humano (14), se ha establecido el análisis de rotación de personal en las áreas de estudio de la compañía, para la identificación del impacto financiero que genera la rotación de personal dentro de la compañía.

La incertidumbre, inestabilidad, genera alta rotación en especial a los nuevos postulantes, y los antiguos trabajadores que réquiem de estabilidad para el cumpliendo de sus objetivos profesionales.

En la rotación de personal la organización debe incurrir en un conjunto de desembolso diarios obligatorios por Ley en la desvinculación del personal saliente demás de los requeridos para el reclutamiento y contratación de quienes cubrirán las vacantes presentadas.

Cabe recalcar que como lo ha considerado el autor (30), al ser una empresa de servicios, la personalización para el cliente juega un papel importante, ya que son los colaboradores de la compañía que darán el servicio requerido por los socios que son clientes frecuentes.

También es importante mantener a los colaboradores motivados y comprometidos con los objetivos de la organización, enfocando las prácticas de recursos humanos en la construcción de la motivación, el compromiso y el desarrollo de las personas en el grupo de talentos, con el fin de que la rotación de personal dentro de la compañía disminuya. Tal como manifiesta Arias Galicia (3) comenta que las organizaciones deben tener la capacidad para el desarrollo, administración de los quipos que posee y emplear el mejoramiento continuo Carvaja (13).

Los costos de la rotación del personal se pueden determinar con el seguimiento al proceso de la contratación, mantenimiento y desvinculación de un colaborador.

Adicional al impacto financiero, también se pueden identificar que la rotación de personal posee impacto en otros factores como el desempeño de los colaboradores y su clima laboral.

Por lo que es importante investigar cómo disminuir la rotación de personal, y motivar a los colaboradores para que continúen en la empresa de una manera comprometida y muy productiva. 
En consecuencia, para los Directivos de la Empresa Multinacional de Venta de Vacaciones Pre Pagadas, es muy importante que se considere el comportamiento de la rotación de personal existente en las áreas operativas y mercadeo, debido a que esto representa un incremento significativo en los costos de la compañía y afectan a la rentabilidad de la misma.

Por lo que el presente trabajo de investigación tiene como objetivo reflejar los costos que actualmente se incurre como resultado de la rotación de personal demostrando que este fenómeno se convierte en un problema de productividad y rentabilidad para la empresa

\subsection{Antecedentes de la Investigación:}

(10), en la investigación Factores que originan la Rotación de Personal en las Empresas Mexicanas, identifican los factores que originan la rotación de personal en las empresas mexicanas; la relación existente entre la rotación y la insatisfacción laboral; los impactos que genera la baja remuneración la selección incorrecta y la poca motivación en la rotación de personal.

Para entender mejor sobre el impacto económico financiero (15) que genera la rotación de personal, realizaremos un análisis de manera independiente, de los factores que intervienen para que se de esta afectación.

Se puede mencionar (16) también que como consecuencia del estudio antes señalado, se existen factores de importancia que motivan a generar la rotación como son:

- La selección incorrecta de personal, es un factor que motiva el aumento de la rotación de personal.

- La baja remuneración incrementa necesidad en el trabajador de buscar otro lugar de trabajo, lo que incide en la rotación del personal.

- La falta de interés del trabajador hace que las capacitaciones no tengan el éxito anhelado.

\section{Materiales y Métodos}

\subsection{Diseño de la Investigación}

Para el desarrollo de la presente investigación se realizará una investigación mixta, basada en los enfoques cuantitativo y cualitativo de acuerdo con lo manifestado por (1). 
Cuantitativo.- con el fin de obtener información que permita identificar los procesos más importantes en la Empresa Multinacional de Venta de Vacaciones Pre pagadas, mediante encuestas basadas en un método inductivo. En la investigación se trata de determinar la correlación entre variables y obtención de resultados objetivos orientado a resultados.

Cualitativo.- permitirá identificar la naturaleza del objeto de estudio y establecer las principales causas del problema Según el autor (2) mediante un método deductivo orientado a los procesos Operativos y Mercadeo de la compañía. La investigación de carácter cualitativo trata los fenómenos que son estudiados mediante técnicas como la observación participante y las entrevistas no estructuradas.

\subsection{Métodos de Investigación}

La presente investigación se realizará mediante técnicas lógicas como son los métodos inductivo y deductivo, razón por la que se utilizara la percepción del problema y un análisis de los datos recopilados para la formación de la hipótesis.

\subsection{Método Inductivo Deductivo}

Parte de casos particulares que pasan a ser de conocimiento general, iniciando por la observación e identificación del problema, que en el caso de la presente investigación serán analizados los procesos de la Gestión de Talento Humano, para determinar las principales causas que generen la Rotación de Personal.

La deducción inicia con la observación de hechos particulares con el propósito de llegar a una conclusión directa. Para esta investigación el método deductivo iniciara con el análisis de todos los costos incurridos por la empresa para el talento humano, para determinar la conclusión final respecto a los que impactan directamente con la Rotación de Personal.

La etapa deductiva, en la cual las generalizaciones logradas mediante la inducción, se usan como premisas para la deducción de enunciados sobre las observaciones iniciales.

\subsection{Población y Muestra}

La población actual (junio) es de 432 colaboradores entre las áreas Operativas y Mercadeo de la compañía, como lo vemos en la tabla 1: 
TABLE 1: Población a investigarse.

AREA
OPERATIVA
MERCADEO

\section{COLABORADORES}

99

333

La muestra es determinada mediante la siguiente formula:

$\mathrm{n}=$ es el número de elementos que conforman la muestra

$\mathrm{N}=$ Población

e = porcentaje de error en los resultados de la investigación social

La muestra fue determinada, a través de la Ec. (1.1).

$$
n=\frac{N}{e^{2}(N-1)+1}
$$

\subsection{Técnicas de Investigación}

\subsubsection{Observación Directa}

Mediante una visita a las áreas Operativas y de Mercadeo de la Empresa Multinacional de Venta de Vacaciones Pre pagadas, serán observados los procesos y el ambiente en el que los colaboradores desarrollan sus actividades laborales (17).

\subsubsection{Encuesta}

Para recopilar información como las necesidades y percepciones del personal, se aplicará una encuesta a la muestra conformada por las áreas Operativas y Mercadeo de la Empresa Multinacional de Venta de Vacaciones Pre pagadas (19).

\subsubsection{Análisis de Documentos}

Se analizará la información cuantitativa de informes y documentos de archivos correspondientes a la empresa, con el fin de analizar los procesos y resultados de la empresa con respecto a la Gestión del Talento Humano y Financiero. (20). 


\subsection{Metodología para el Tratamiento de Información}

Se empleará la técnica de encuesta a la población conformada por el personal que es parte de áreas Operativas y Mercadeo.

Las fuentes secundarias serán utilizadas mediante el análisis de la diferente documentación de archivos y bibliografía del tema de estudio (21).

Los resultados de la información procesada serán demostrados mediante la utilización de tablas resumen y cuadros estadísticos, estableciendo relación entre las variables objeto de estudio.

\subsection{Sistema de Variables}

Según (4) en investigación existen dos tipos de variables:

\subsubsection{Variable Independiente}

Se considera como la característica que enfoca la causa que genera a la variable dependiente en el fenómeno estudiado.

Para la investigación se ha determinado como variable independiente a la "Rotación de Personal"

En esta variable podrá ser analizado las siguientes dimensiones como: contratación, motivación y desvinculación.

\subsubsection{Variable Dependiente}

Es considerado como el efecto que se genera después del tratamiento en la variable independiente que requiere una medición.

En este caso la variable dependiente será el resultado obtenido del análisis del "Impacto Financiero"

\subsubsection{Variable Moderadora}

Este tipo de variable se considera como de carácter cualitativo o cuantitativo que genera un efecto en la magnitud de la relación que existe entre una variable independiente o de perfil predictorio y la variable dependiente o generadora de criterio, los efectos que se generan se denominan como los de interacción (12). 
En el desarrollo de la investigación este tipo de variable se conforma de las subvariables representadas por la Edad Sexo Función tiempo de servicio del encuestado (12).

\subsubsection{Variable de Confusión}

Se considera como la tercera variable con carácter de mediadora que impacta de forma negativa a la relación que se genera entre la variable independiente y la dependiente, provocando en el investigador un análisis equivocado de los resultados mostrando la presencia de una correlación que se denomina como falsa produciendo un rechazo de un inadecuado de la hipótesis nula presentada (9).

En el desarrollo de la investigación este tipo de variable se identifica en las subvariables relacionadas con:

\subsubsection{Operacionalización de las Variables}

\subsubsection{Validez y Confiabilidad de Instrumentos}

\section{Validez del instrumento de investigación}

Según (7) los expertos pueden validar las diferencias de las categorías y si se cumplen, basándose en los criterios expuestos por Moriyama como guía para la construcción de cuestionarios y que son:

1. Razonable y comprensible: claro entendimiento de las diferentes preguntas que evalúan el factor que se pretende medir.

2. Sensible a variaciones en el factor a medir.

3. Suposiciones justificables y razonables: validar si se justifica la utilización de cada una de las preguntas detalladas en el cuestionario.

4. Componentes claramente definidos: confirmar si cada pregunta está definida claramente con el tema.

Las herramientas de investigación que serán aplicadas en esta investigación, serán validadas por jefes de área a quienes se consideran expertos en los temas a indagar, en este caso la investigación está dirigida al personal de Mercadeo y Operaciones de la empresa. 


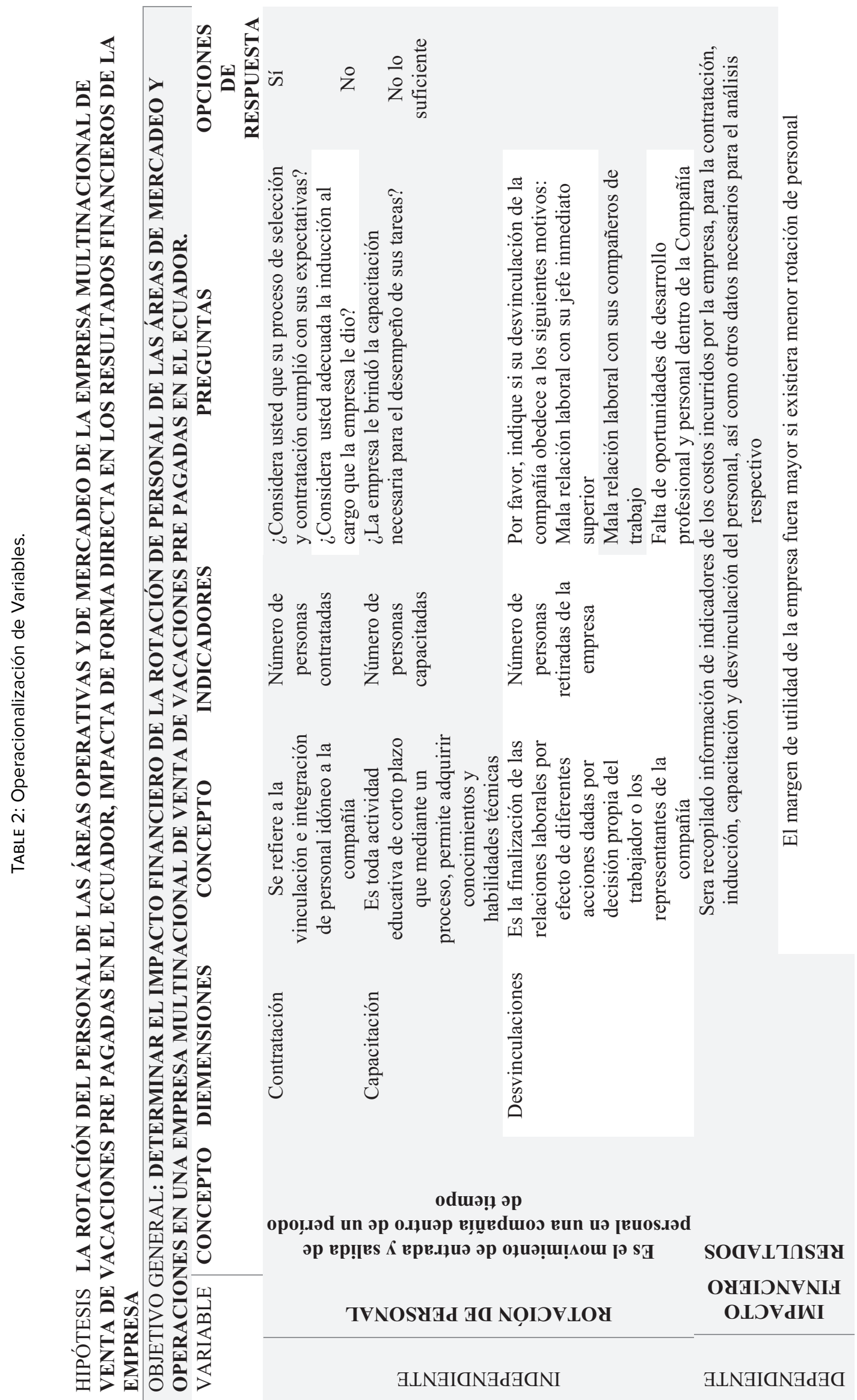




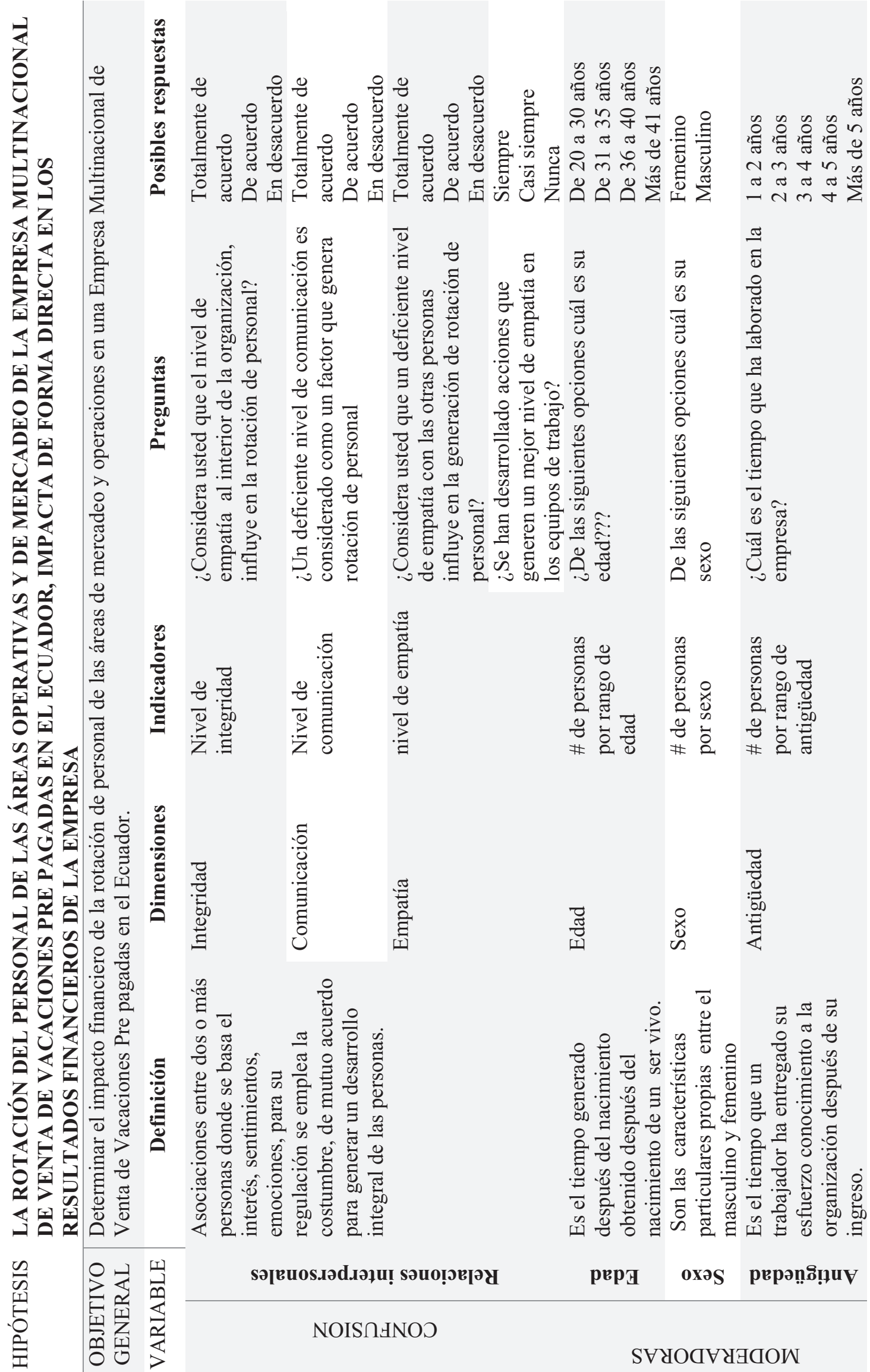




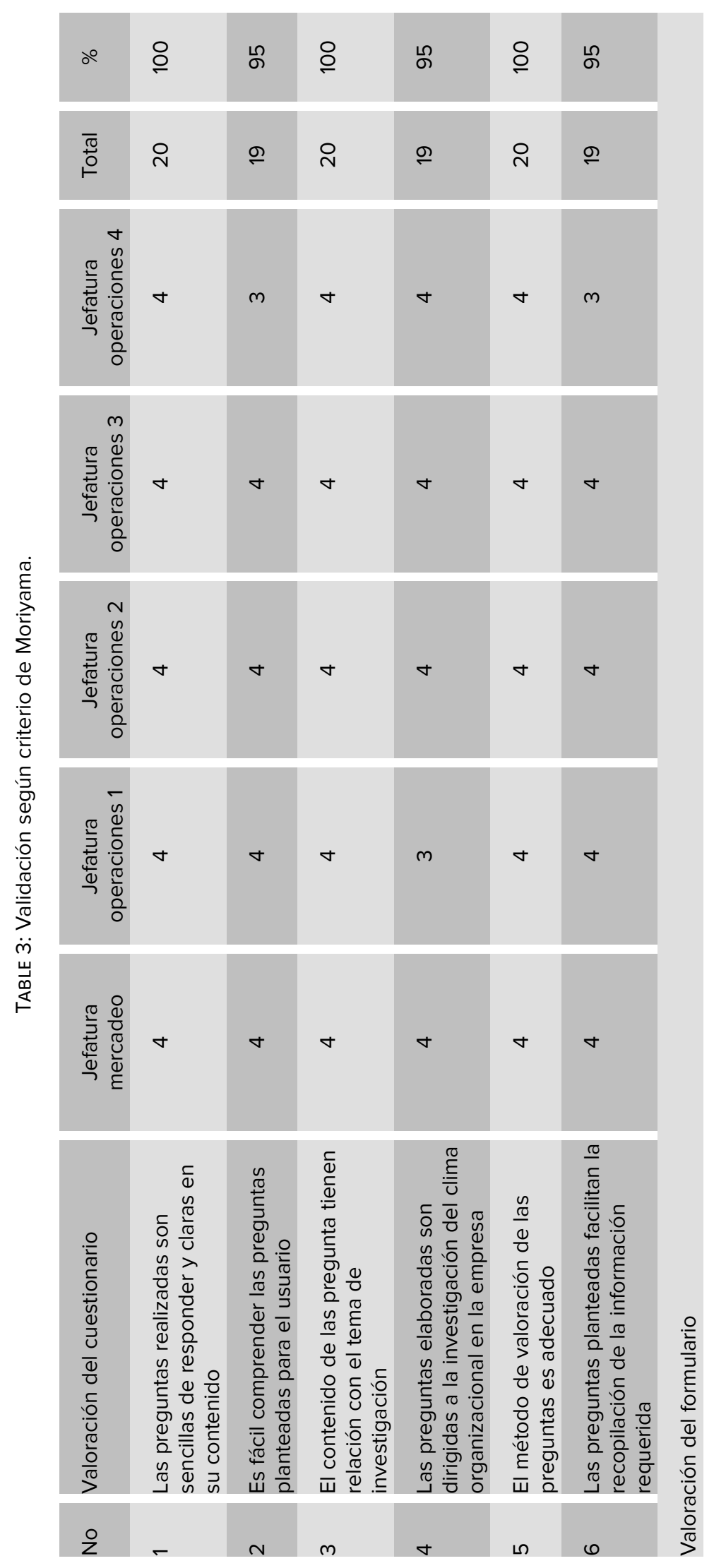




\section{Confiabilidad}

Para validar la confiabilidad de los elementos a utilizar en la encuesta se utilizará el método Alfa de Cronbach, que es un instrumento estadístico que emplea un conjunto de preguntas que buscan una adecuada medición de carácter investigativo. (9)

El índice de Cronbach se calcula a través de la fórmula 1.2:

Dónde:

a = índice

$\mathrm{Si}=$ es la varianza de cada pregunta

St $=$ es la varianza de los valores totales

$\mathrm{K}=$ es el número de preguntas

$$
a=\frac{K}{K-1}\left[1 \frac{\Sigma s^{2}}{S_{T}^{2}}\right]
$$

Se sugiere las siguientes recomendaciones para evaluar los coeficientes de alfa de Cronbach:

- Coeficiente alfa $>9$ es excelente

- Coeficiente alfa $>8$ y $<9$ es bueno

- Coeficiente alfa $>7$ y $<8$ es aceptable

- Coeficiente alfa $>6$ y $<7$ es cuestionable

- Coeficiente alfa $>5$ y $<6$ es pobre

- Coeficiente alfa $<5$ es inaceptable

El coeficiente permite realizar una matriz de correlaciones cuadradas y en las intersecciones los valores de los coeficientes que se generan entre las variables detalladas en filas y las variables en columnas, la correlación entre cada dos variables que forman un triángulo de variables duplicadas en la parte superior e inferior. Al coincidir las variables el valor del coeficiente será igual a 1.

Después del tratamiento de las preguntas que son parte del instrumento que se aplicó a los colaboradores entre las áreas Operativas y Mercadeo de la compañía se ha determinado que el cálculo del Alfa de Cronbach se estructura de la siguiente forma:

\section{Escala: Alfa de Cronbach}

Tras la aplicación del instrumento se muestra que el resultado tiene tendencia a 1 por lo cual tiene confiabilidad aceptable. 
TABLE 4: Resumen del procesamiento de los casos.

\begin{tabular}{l|l|c|c|}
\hline & & N & $\%$ \\
Casos & Válidos & 204 & 96,2 \\
& Excluidos $^{a}$ & 8 & 3,8 \\
& Total & 212 & 100,0 \\
\hline a. Eliminación por lista basada en todas las variables del procedimiento.
\end{tabular}

TABLE 5: Valor calculo Alfa de Cronbach.

Alfa de Cronbach

0,772
$\mathrm{N}$ elementos

8

\section{Discusión de Resultados}

\subsection{Análisis y Resultados}

\subsubsection{Procesamiento de información}

\section{1. ¿Considera usted que su proceso de selección y contratación cumplió con sus expectativas?}

TABLE 6: Proceso de selección y contratación cumple con expectativas.

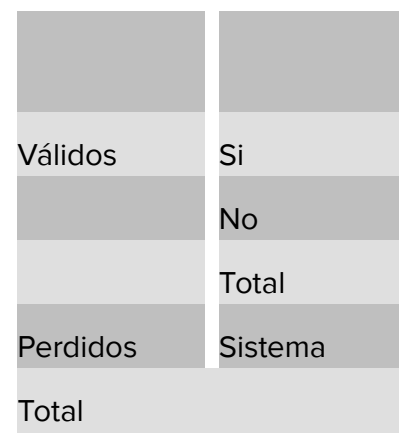

\begin{tabular}{|c|}
\hline Frecuencia \\
\hline 175 \\
\hline 32 \\
\hline 207 \\
\hline 5 \\
\hline 212 \\
\hline
\end{tabular}

\begin{tabular}{|c|}
\hline Porcentaje \\
\hline 82,5 \\
\hline 15,1 \\
\hline 97,6 \\
\hline 2,4 \\
\hline 100,0 \\
\hline
\end{tabular}

\begin{tabular}{|c|}
\hline $\begin{array}{c}\text { Porcentaje } \\
\text { válido }\end{array}$ \\
\hline 84,5 \\
\hline 15,5 \\
\hline 100,0 \\
\hline
\end{tabular}
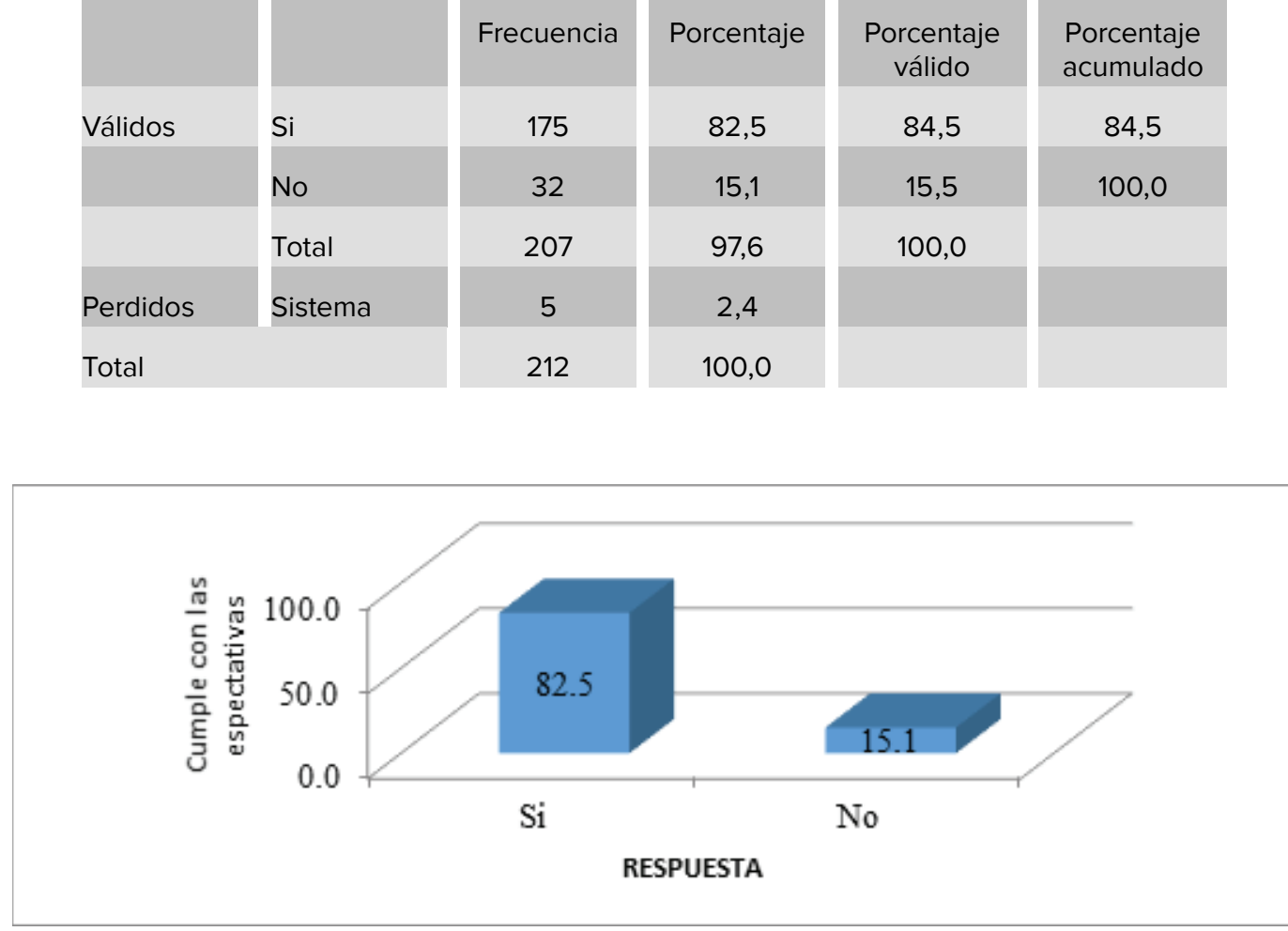

Figure 1: Proceso de selección y contratación cumple con expectativas. (Fuente: Investigación de campo). 
Análisis: Tras el procesamiento de la información que se obtuvo de la muestra del personal al levantar información sobre el proceso de selección y contratación se ha identificado que para el $82,5 \%$ si cumplió sus expectativas y para el $15,1 \%$ no lleno sus expectativas para el proceso de contratación.

Interpretación: Al analizar la información obtenida se ha identificado que la mayor parte de personal para el proceso de inducción y selección si cumplió con sus expectativas.

\section{2. ¿Considera usted adecuada la inducción al cargo que la empresa le dio?}

TABLE 7: Considera usted adecuada la inducción al cargo que la empresa le dio.

\begin{tabular}{|c|c|c|c|c|c|}
\hline & & Frecuencia & Porcentaje & $\begin{array}{l}\text { Porcentaje } \\
\text { válido }\end{array}$ & $\begin{array}{l}\text { Porcentaje } \\
\text { acumulado }\end{array}$ \\
\hline \multirow[t]{4}{*}{ Válidos } & $\mathrm{Si}$ & 166 & 78,3 & 80,2 & 80,2 \\
\hline & No & 29 & 13,7 & 14,0 & 94,2 \\
\hline & $\begin{array}{l}\text { No lo } \\
\text { suficiente }\end{array}$ & 12 & 5,7 & 5,8 & 100,0 \\
\hline & Total & 207 & 97,6 & 100,0 & \\
\hline Perdidos & Sistema & 5 & 2,4 & & \\
\hline Total & & 212 & 100,0 & & \\
\hline
\end{tabular}

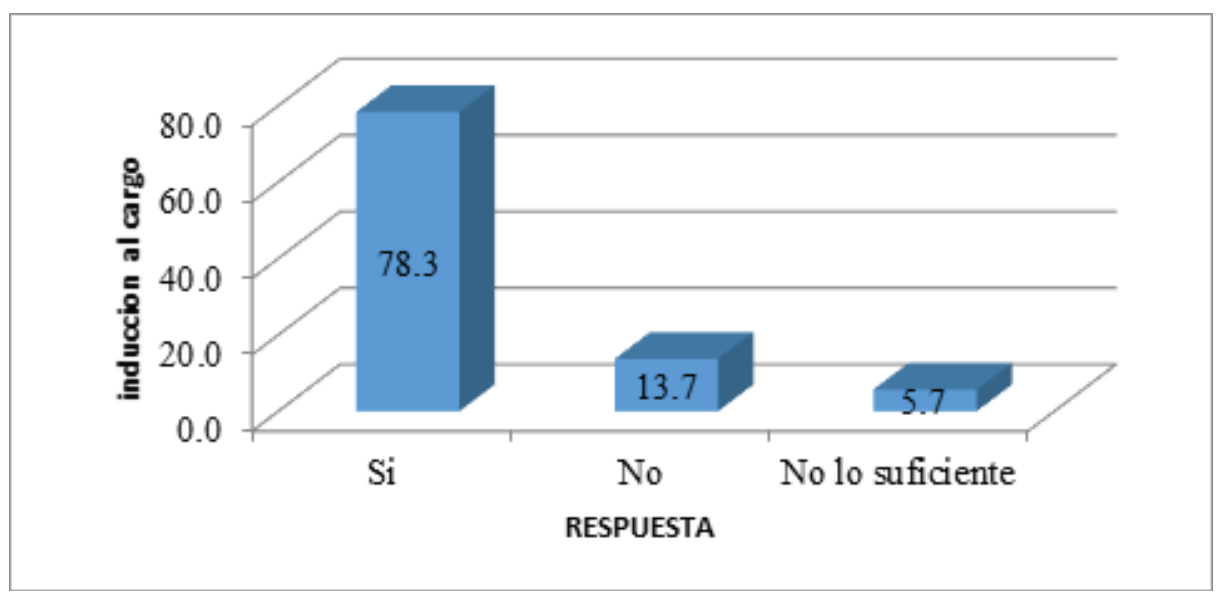

Figure 2: Considera usted adecuada la inducción al cargo que la empresa le dio. (Fuente: Investigación de campo).

Análisis: Tras el procesamiento de la información que se obtuvo de la muestra del personal se muestra que el 78,3 \% comenta que si es adecuada la inducción al cargo que la empresa le dio, mientras que el 13,7 \% señalan que no es adecuado.

Interpretación: Con la información obtenida se ha identificado que la mayor parte de personal señala que la inducción al cargo que la empresa le dio poniendo en evidencia 
que al nuevo personal que ingresa si se le entrego la respectiva información sobre las funciones que debe cumplir como parte de sus responsabilidades.

\section{3. ¿La empresa le brindó la capacitación necesaria para el desempeño de sus} tareas?

TABLE 8: La empresa le entrega capacitación necesaria.

\begin{tabular}{|c|c|c|c|c|c|}
\hline & & Frecuencia & Porcentaje & $\begin{array}{l}\text { Porcentaje } \\
\text { válido }\end{array}$ & $\begin{array}{l}\text { Porcentaje } \\
\text { acumulado }\end{array}$ \\
\hline \multirow[t]{4}{*}{ Válidos } & $\mathrm{Si}$ & 166 & 78,3 & 80,2 & 80,2 \\
\hline & No & 29 & 13,7 & 14,0 & 94,2 \\
\hline & No lo suficiente & 12 & 5,7 & 5,8 & 100,0 \\
\hline & Total & 207 & 97,6 & 100,0 & \\
\hline Perdidos & Sistema & 5 & 2,4 & & \\
\hline Total & & 212 & 100,0 & & \\
\hline
\end{tabular}

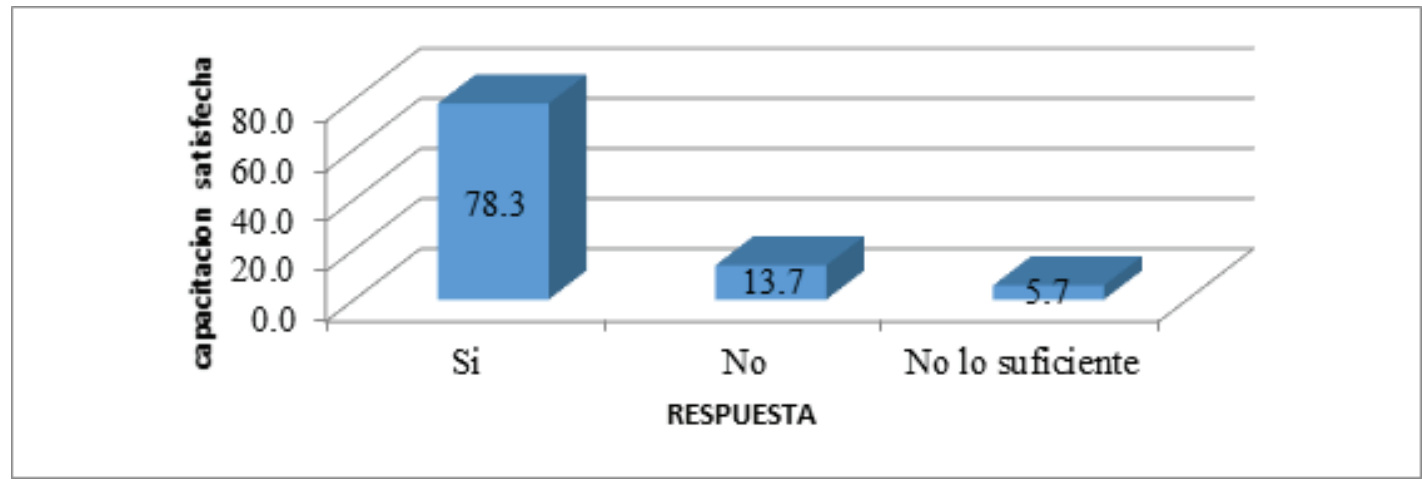

Figure 3: La empresa le entrega capacitación necesaria. (Fuente: Investigación de campo).

Análisis: Tras el procesamiento de la información que se obtuvo de la muestra del personal al levantar información sobre la capacitación recibida que para el 78,3 \% la empresa le entrego la capacitación necesaria y para el 13,7 \% no para ellos les pareció inconclusas.

Interpretación: Se ha identificado que la mayor parte de personal si obtuvo la capacitación necesaria y las personas que no puede deberse a una falta de interés en las capacitaciones posible razón para no obtener el éxito anhelado.

\section{Por favor, indique si su desvinculación de la compañía obedece a los siguientes} motivos:

Por la remuneración mensual recibida

Análisis: Tras el procesamiento de la información que se obtuvo de la muestra del personal al levantar información, se ha identificado que para el 88,7 \% no está conforme con la remuneración y para el 9 \% si está de acuerdo con la remuneración. 
TABLE 9: Remuneración recibida.

\begin{tabular}{|l|c|c|c|c|c|}
\hline & & Frecuencia & Porcentaje & $\begin{array}{c}\text { Porcentaje } \\
\text { válido }\end{array}$ & $\begin{array}{c}\text { Porcentaje } \\
\text { acumulado }\end{array}$ \\
\hline Válidos & Si & 19 & 9,0 & 9,2 & 9,2 \\
\hline & No & 188 & 88,7 & 90,8 & 100,0 \\
\hline & Total & 207 & 97,6 & 100,0 & \\
\hline Perdidos & Sistema & 5 & 2,4 & & \\
\hline Total & & 212 & 100,0 & & \\
\hline
\end{tabular}

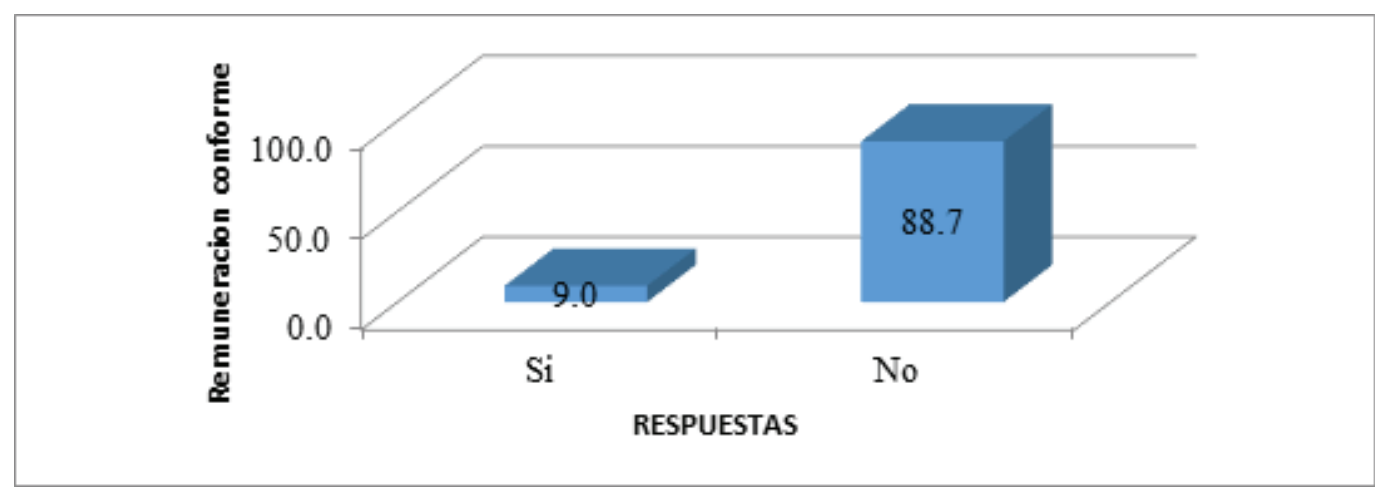

Figure 4: Remuneración recibida. (Fuente: Investigación de campo).

Interpretación: Al analizar la información obtenida se ha identificado que la mayor parte de personal no está de acuerdo debido a la baja remuneración recibida.

\section{Mala relación laboral con su jefe inmediato superior}

TABLE 10: Mala relación laboral jefe inmediato.

\begin{tabular}{|l|l|c|c|c|c|}
\hline & Frecuencia & Porcentaje & $\begin{array}{c}\text { Porcentaje } \\
\text { válido }\end{array}$ & $\begin{array}{c}\text { Porcentaje } \\
\text { acumulado }\end{array}$ \\
\hline Válidos & $\mathrm{Si}$ & 12 & 5,7 & 5,8 & 5,8 \\
\hline & No & 194 & 91,5 & 94,2 & 100,0 \\
\hline & Total & 206 & 97,2 & 100,0 & \\
\hline Perdidos & Sistema & 6 & 2,8 & & \\
\hline Total & & 212 & 100,0 & & \\
\hline
\end{tabular}

Análisis: Tras el procesamiento de la información que se obtuvo de la muestra del personal al levantar información se ha identificado que para el 91,5 \% no tiene una mala relación con su jefe y para el 5,7 \% sí.

Interpretación: Se ha evidenciado que la mayor parte de personal se siente motivado con el liderazgo de su jefe inmediato.

Mala relación laboral con sus compañeros de trabajo 


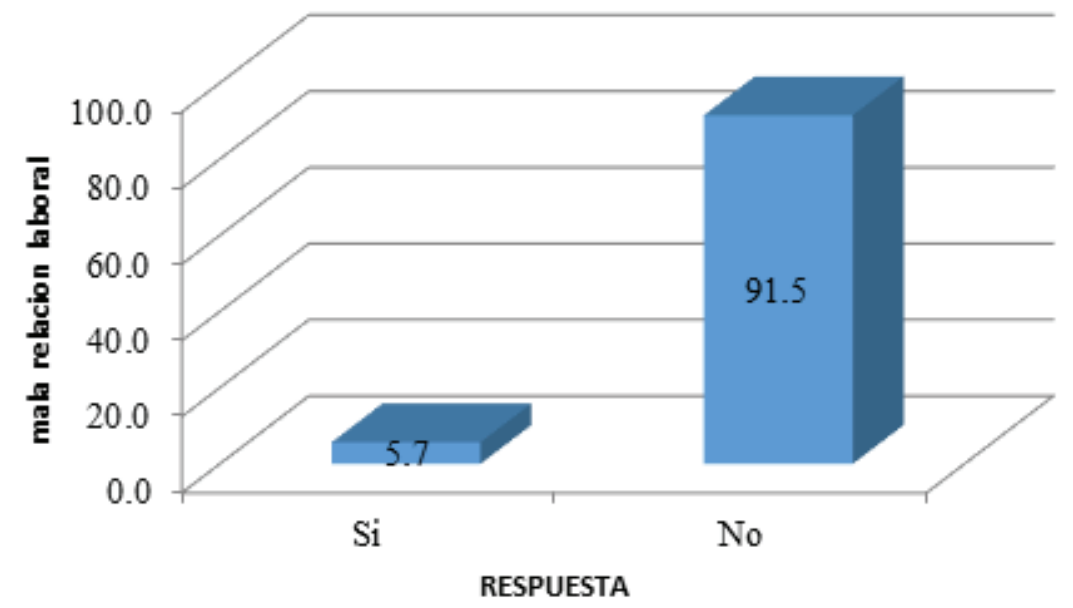

Figure 5: Mala relación laboral jefe inmediato. (Fuente: Investigación de campo).

TABLE 11: Mala relación laboral con sus compañeros.

\begin{tabular}{|l|l|c|c|c|c|}
\hline & & Frecuencia & Porcentaje & $\begin{array}{c}\text { Porcentaje } \\
\text { válido }\end{array}$ & $\begin{array}{c}\text { Porcentaje } \\
\text { acumulado }\end{array}$ \\
\hline Válidos & Si & 10 & 4,7 & 4,9 & 4,9 \\
\hline & No & 196 & 92,5 & 95,1 & 100,0 \\
\hline & Total & 206 & 97,2 & 100,0 & \\
\hline Perdidos & Sistema & 6 & 2,8 & & \\
\hline Total & & 212 & 100,0 & & \\
\hline
\end{tabular}

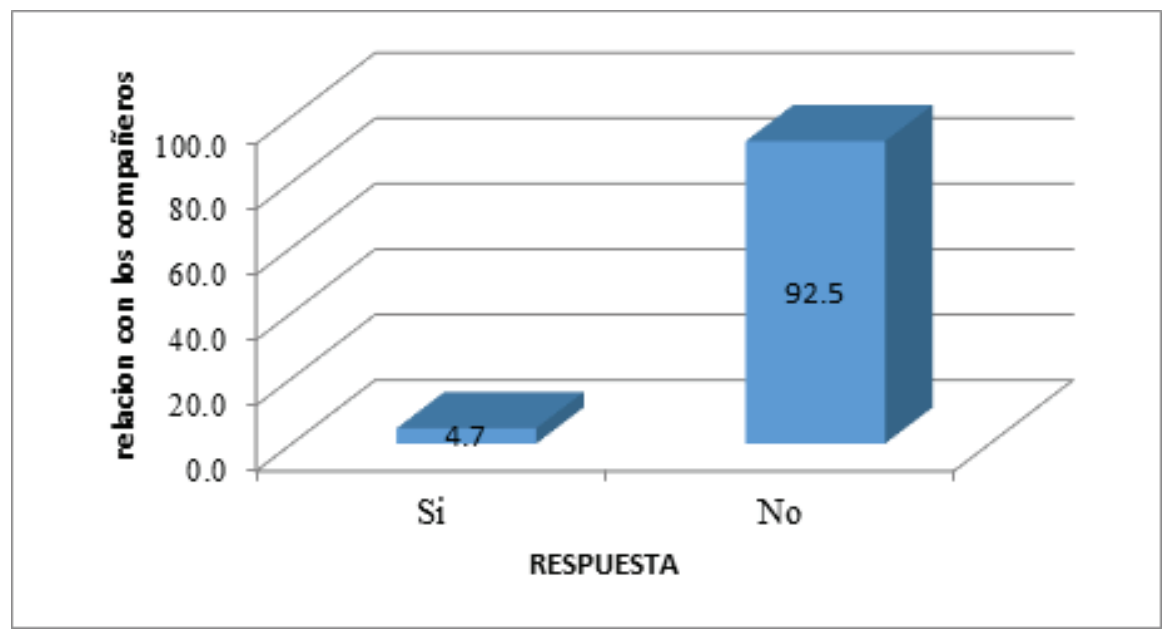

Figure 6: Representación gráfica de lamala relación laboral con sus compañeros.

Análisis: Tras el procesamiento de la información que se obtuvo de la muestra del personal al levantar información se ha identificado que el 92,5 \% no tiene una mala relación entre compañeros de trabajo, el tipo de relaciones humanas dentro de la 
organización y el ambiente laboral es agradable para el 4,7 \% si es mala su relación laboral entre compañeros.

Interpretación: Al verificar la información obtenida se ha identificado que la mayor parte de personal tiene una buena relación laboral formándose un ambiente agradable con buenas condiciones para formar un buen equipo de trabajo.

Falta de oportunidades de desarrollo profesional y personal dentro de la Compañía

TABLE 12: Falta de oportunidades de desarrollo en la empresa.

\begin{tabular}{|l|l|c|c|c|c|}
\hline & Frecuencia & Porcentaje & $\begin{array}{c}\text { Porcentaje } \\
\text { válido }\end{array}$ & $\begin{array}{c}\text { Porcentaje } \\
\text { acumulado }\end{array}$ \\
\hline Válidos & $\mathrm{Si}$ & 2 & 0,9 & 1,0 & 1,0 \\
\hline & No & 205 & 96,7 & 99,0 & 100,0 \\
\hline & Total & 207 & 97,6 & 100,0 & \\
\hline Perdidos & Sistema & 5 & 2,4 & & \\
\hline Total & & 212 & 100,0 & & \\
\hline
\end{tabular}

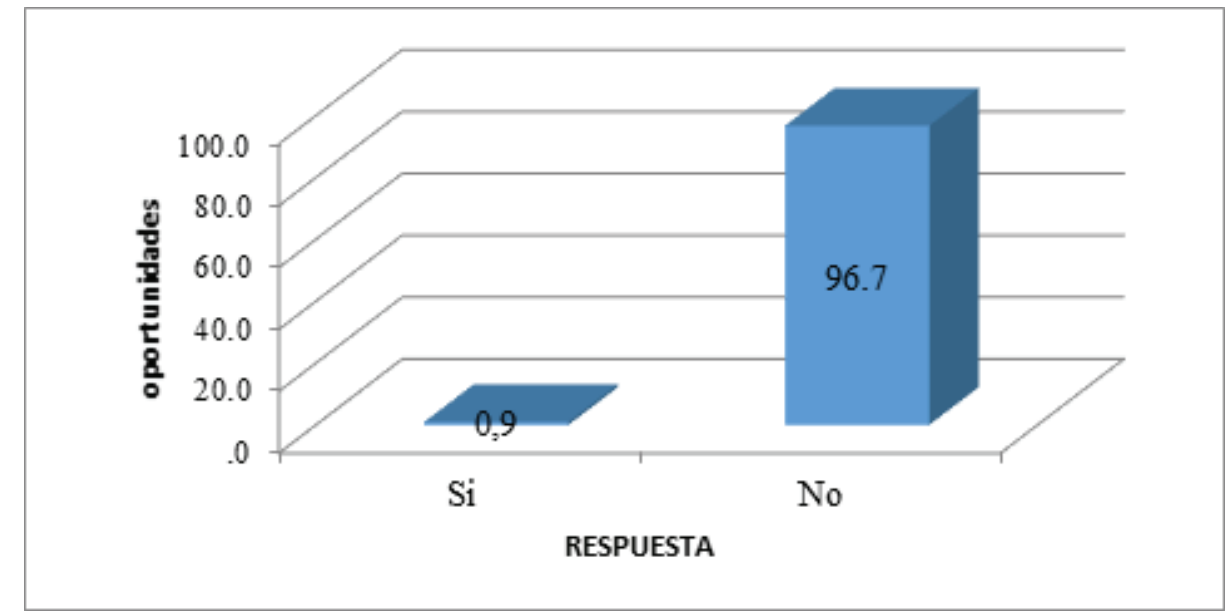

Figure 7: Falta de oportunidades de desarrollo en la empresa. (Fuente: Investigación de campo).

Análisis: Tras el procesamiento de la información que se obtuvo de la muestra del personal al levantar información se ha identificado que para el 96,7 \% la falta de oportunidades dentro de la empresa no afecta en su desarrollo laboral y para el 0,9\% si siente la falta de oportunidades.

Interpretación: Se ha identificado que no existe la falta de oportunidades en el crecimiento profesional de la empresa y no afecta este factor en la organización. 


\subsubsection{Análisis del impacto financiero en relación a la Rotación de Per- sonal}

Según la información proporcionada por el área financiera de la Empresa Multinacional de Venta de Vacaciones Pre pagadas se va a presentar un informe sobre el comportamiento de los costos primarios, secundarios, terciarios que se generan sobre la presencia de la rotación de personal en un periodo comprendido entre 2012 y marzo del 2014 los cuales se muestran de la siguiente forma:

\section{Costos primarios de rotación de personal}

- Reclutamiento y selección

- Registro y documentación

- Costos de ingreso

- Costos de desvinculación

\section{Costos secundarios de rotación de personal}

- Efectos en las operaciones

- Efectos en la actitud del personal

\section{Costos Terciarios de rotación de personal}

- Costos de inversión extra

\section{Balance de Ingresos y Gastos 2012 -- 2014}

Al analizar el comportamiento del balance de ingresos y gastos en el periodo 2012 -2014, de la Empresa Multinacional de Venta de Vacaciones Prepagadas sucursal Quito se ha identificado que se existe un incremento en el volumen de ingresos, además de los egresos por lo cual se ha identificado que para el año 2012 la utilidad operativa es negativa porque el volumen de egresos es mayor sobre los ingresos.

En el año 2013 y el 2014 se ha identificado que ya existe la presencia de un volumen de utilidad razonable donde se muestra que la organización logro cumplir sus objetivos esperados además se debe comentar que en el año 2012 los costos de personal tienen una representatividad del 60,2 \% para el 2013 es de 65,71\% y en el 2014 es de 67,79 $\%$. 


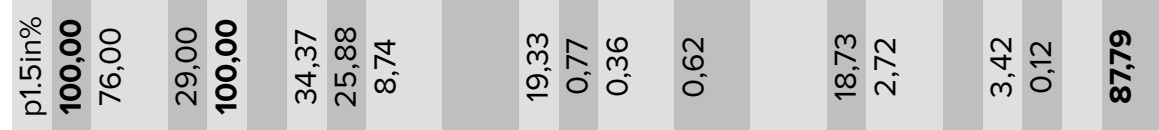

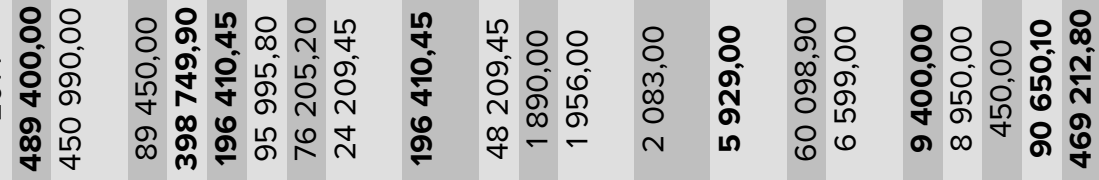

응요 으

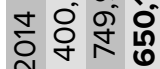
尚

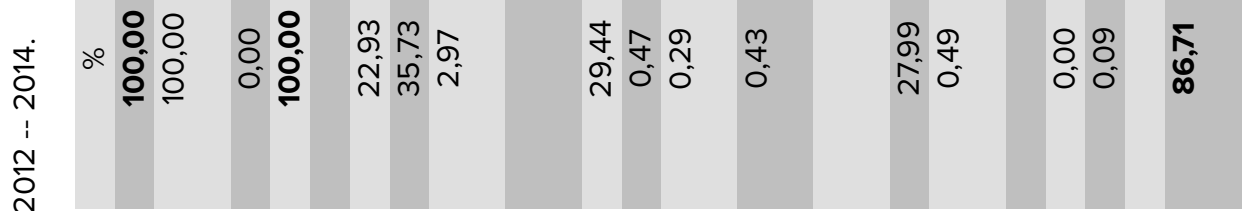

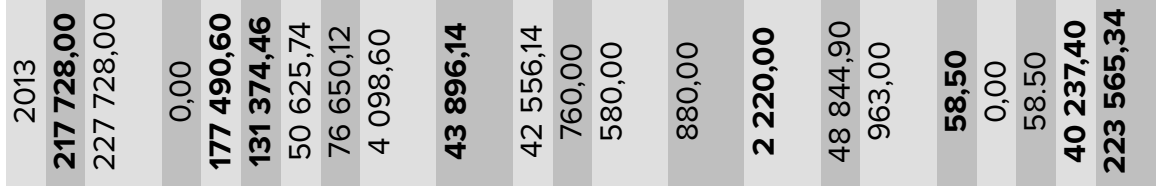
웅ㅇㅇ

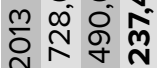
กิ

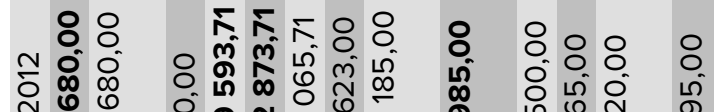

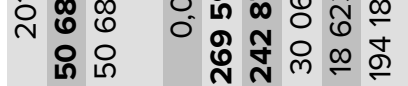




\section{Resumen de la plantilla}

Para el año 2012 se muestra que el grupo de colaboradores al inicio del periodo se conformó por 35 trabajadores en la sucursal de la ciudad de Quito conformado por 1 directivo, 2 administrativos, 12 colaboradores en el área de operación, 20 en el área de mercadeo donde al final del año existen 35 colaboradores evidenciando que no existe rotación de personal en ese año y ausencia de impacto negativo en la gestión financiera.

En el año 2013 se ha identificado que el grupo al inicio del periodo concentro a 35 colaboradores donde se registró 512 ingresos de nuevo personal, de la siguiente forma 180 personas en el área de operaciones, 332 en el área de mercadeo donde tras la finalización del año se muestra que el mismo personal que ingreso al final del año tuvo que salir de la organización poniendo en evidencia ya la presencia de rotación de personal.

En el año 2014 se muestra que el grupo de colaboradores al inicio del periodo fue de 35 colaboradores a través de la siguiente distribución 1 directivo, 2 administrativos, 12 colaboradores en el área de operación, 20 en el área de mercadeo además se identificó que existieron 960 nuevos ingresos donde 250 en el área de operación, 710 en mercadeo tras la finalización del año se muestra que el personal que ingreso al final tuvo que salir.

Al analizar el nivel de nuevos ingresos de personal, salidas en el periodo 2012- 2014 se muestra que a partir del 2013 ya existe la presencia de rotación de personal de igual forma para el año 2014 se incrementa esta tendencia lo cual es evidente que se desarrollen acciones para controlar las cifras de crecimiento de rotación de personal en la organización en el año 2012.

\section{Motivos de salida}

Tras la identificación de las tendencias de comportamiento de la rotación de personal al interior de la Empresa Multinacional de Venta de Vacaciones Pre pagadas sucursal Quito se va a presentar los motivos que generaron la salida del personal.

Al analizar los factores que han generado la rotación de personal entre el periodo 2012-2014 se muestra que:

- En el año 2013, 198 personas salieron por terminación de contrato, 223 por mejores condiciones salariales, 90 por visto bueno, 1 por incapacidad por lo cual la cifra total es de 512 personas. 
TABLE 14: Motivos de salida personal 2012 -- 2014.

Motivos de salida
Terminación de Contrato
Mejores Condiciones
Salariales
Muerte
Visto Bueno
Enfermedad
Incapacidad
Jubilación
Otros (Problemas Familiares)
Total

2012
0
0
0
0
0
0
0
0
0

2013
198
223
0
90
0
1
0
0
$\mathbf{5 1 2}$

\begin{tabular}{|c|}
\hline 2014 \\
\hline 250 \\
700 \\
\hline 0 \\
\hline 8 \\
\hline 0 \\
\hline 0 \\
\hline 0 \\
2 \\
\hline 960
\end{tabular}

Fuente: Investigación Documental.

- Para el año 2014, 250 colaboradores abandonaron la organización por terminación de contrato, 700 personas por la presencia de mejores condiciones salariales, 8 colaboradores como resultado del uso del visto bueno y 2 por otros problemas ya sean personales y familiares.

\section{Costos generados por la rotación de personal}

Según la información obtenida a través de la tabla se muestra que en el año 2012 los costos primarios de la gestión del personal represento un valor de USD 3 050,00 donde abarca a los desembolsos desarrollados por concepto de reclutamiento y selección; registro y documentación; valores por ingreso de nuevo personal pero aún no se enfoca en los de rotación de personal porque aún no se registra este tipo de comportamiento.

Para el año 2013 se muestra que ya evidencia la presencia de rotación de personal donde sus costos equivalen a un valor de USD 50 450,72 donde se evidencia que los costos primarios por concepto de reclutamiento y selección; registro y documentación; valores por ingreso de nuevo personal tiene un valor de USD 26 450,72 con relación a los Costos secundarios su valor es de USD 18 000,00 y los terciarios de rotación de personal contempla un valor de USD 6 000,00 considerándose como un valor de inversión extra.

De igual forma para el año 2014 se ha identificado que los costos que la presencia de la rotación de personal genera tiene un valor de USD 103 165,50 donde los costos primarios de rotación de personal tienen un valor de USD 47 615,50 conformado 
TABLE 15: Costos generados por la rotación de personal2012 -- 2014

Costos
Costos Primarios De Rotación De
Personal
Reclutamiento y Selección
Registro y Documentación
Costos de Ingreso
Costos de Desvinculación
Costos Secundarios de Rotación de
Personal
Efectos En Las Operaciones
Efectos En La Actitud Del Personal
Costos Terciarios De Rotación De
Personal
Costos De Inversión Extra
TOTAL

\begin{tabular}{l}
2012 \\
3050,00 \\
400,00 \\
90,00 \\
2560,00 \\
0,00 \\
0,00 \\
0,00 \\
0,00 \\
0,00 \\
\hline 0,00 \\
\hline $\mathbf{3} 050,00$
\end{tabular}

\begin{tabular}{|c|c|}
\hline 2013 \\
\hline 26450,72 \\
\hline 3500,00 \\
\hline 8000,00 \\
\hline 5500,00 \\
\hline 9450,72 \\
\hline 18000,00 \\
\hline 10000,00 \\
\hline 8000,00 \\
\hline 6000,00 \\
\hline 6000,00 \\
\hline 50450,72 \\
\hline
\end{tabular}

\begin{tabular}{|c|c|}
\hline \multicolumn{1}{|c|}{2014} \\
\hline 47615,50 \\
\hline 9500,00 \\
\hline 10450,00 \\
\hline 20245,50 \\
\hline 44550,00 \\
\hline 36500,00 \\
\hline 8050,00 \\
\hline 11000,00 \\
\hline 11000,00 \\
\hline 103165,50 \\
\hline
\end{tabular}

Fuente: Investigación Documental.

TABLE 16: Costos generales Empresa sucursal Quito 2012-2014.

\begin{tabular}{|l|c|c|c|}
\hline RUBROS & $\mathbf{2 0 1 2}$ & $\mathbf{2 0 1 3}$ & $\mathbf{2 0 1 4}$ \\
\hline Ingresos & 50680,00 & 217728,00 & 489400,00 \\
\hline Egresos & 3050,00 & 50450,72 & 103165,50 \\
\hline Total & $\mathbf{4 7 6 3 0 , 0 0}$ & $\mathbf{1 6 7 2 7 7 , 2 8}$ & $\mathbf{3 8 6} \mathbf{2 3 4 , 5 0}$ \\
\hline
\end{tabular}

Fuente. Investigación Documental.

Elaborado por: Autores.

por el concepto de reclutamiento y selección; registro y documentación; valores por ingreso de nuevo personal, posteriormente existen los costos secundarios de rotación de personal donde se muestra a los efectos en las operaciones, actitud del personal por un valor de USD 44550,00 y finalmente se muestra a los Costos Terciarios con un valor de USD 103 165,50 donde tiene representación de la inversión extra.

A continuación, se muestra el comportamiento de los costos en el periodo 2012-2014 donde se refleja el incremento de los costos a través de la siguiente tabla:

En el año 2012 se muestra que no existe rotación de personal por ser el punto de partida de la evaluación, a partir del año 2013, se muestra un incremento de los costos generados por la presencia de rotación de personal representado por un valor de 11,47 $\%$ donde se considera como un incremento significativo pero a la vez genera una alta preocupación. Para el año 2014 se evidencia que se ha generado un incremento del 
TABLE 17: Comportamiento de los costos en el periodo 2012-2014.

\begin{tabular}{|l|c|c|c|c|}
\hline Año & Valor al inicial & Valor al final & $\%$ incremento & $\begin{array}{c}\text { Aspecto } \\
\text { relevante }\end{array}$ \\
\hline $\mathbf{2 0 1 2}$ & - & 3050,00 & - & $\begin{array}{c}\text { Periodo de } \\
\text { evaluación }\end{array}$ \\
\hline $\mathbf{2 0 1 3}$ & 3050,00 & 50450,72 & $11,47 \%$ & Leve incremento \\
\hline $\mathbf{2 0 1 4}$ & 50450,72 & 103165,50 & $48,90 \%$ & Alto incremento \\
\hline
\end{tabular}

Fuente: Investigación Documental.

$48,90 \%$ lo cual pone en evidencia que existe un alto incremento de los costos como resultado del uso de mejores condiciones salariales aplicadas en el 2014.

A continuación se muestra los valores de carácter individual que se generaron en un periodo de un ano donde no se evidencia la presencia de rotación de personal para luego evidenciar los respectivos cambios que se han ejecutado en la Empresa Multinacional de Venta de Vacaciones Pre pagadas sucursal Quito originando un alto impacto.

\section{Conclusiones}

Como conclusiones y que la investigación arrojo se especifican a través del análisis y comparación de las preguntas establecidas, objetivos específicos y las conclusiones del resultado donde se presenta los siguientes resultados:

Al analizar, tratar la información obtenida se ha identificado que la mayor parte de personal no está de acuerdo debido a la baja remuneración recibida. La rotación de personal la organización debe incurrir en un conjunto de desembolso diarios obligatorios por Ley en la desvinculación del personal saliente demás de los requeridos para el reclutamiento y contratación de quienes cubrirán las vacantes presentadas.

Se maneja un mejor sueldo monetario y no monetario, existe calidad del clima laboral, manejan niveles de comunicación eficiente, la flexibilidad laboral es manejada adecuadamente con el personal, alineación al estilo de liderazgo.

\section{References}

[1] Albán Villacís, J. (Mayo de 2012). Metodología de la Investigación. Quito, Pichincha, Ecuador.

[2] Arboleda, C. (11 de Junio de 2012). Programa de Maestría de Desarrollode Talento Humano. Análisis comparativo de la Evaluación de Potencial con otros sistemas de 


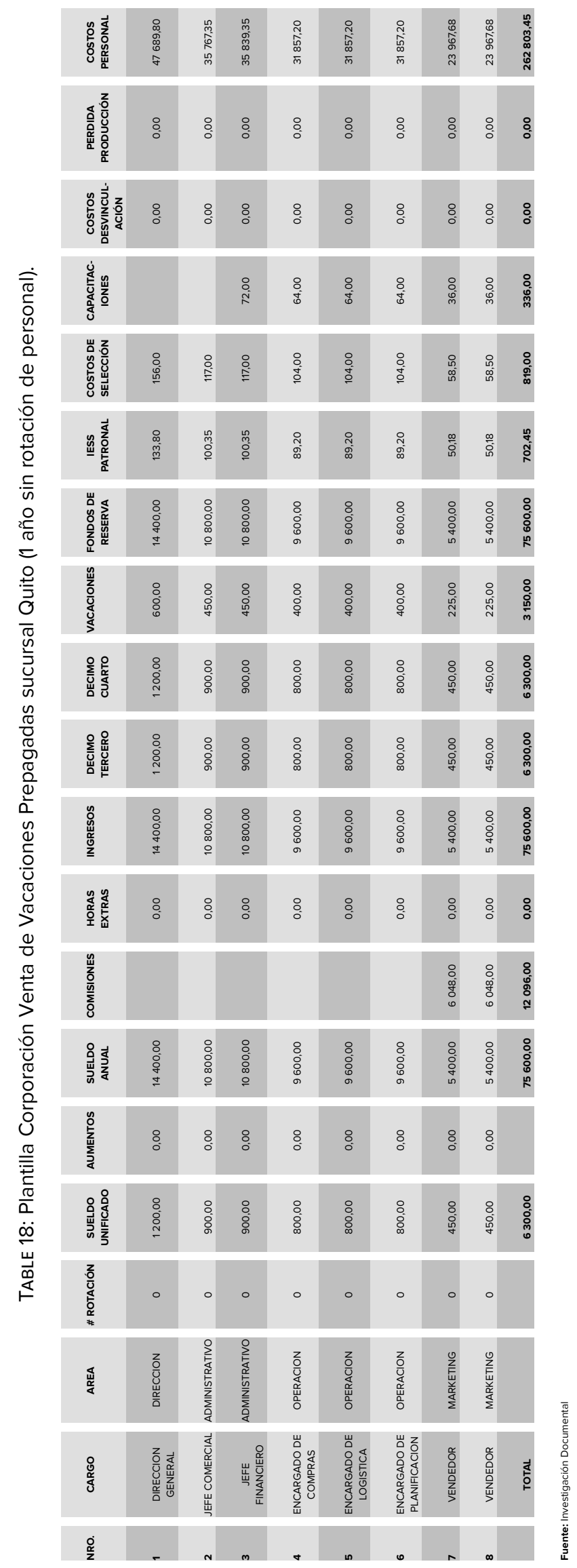




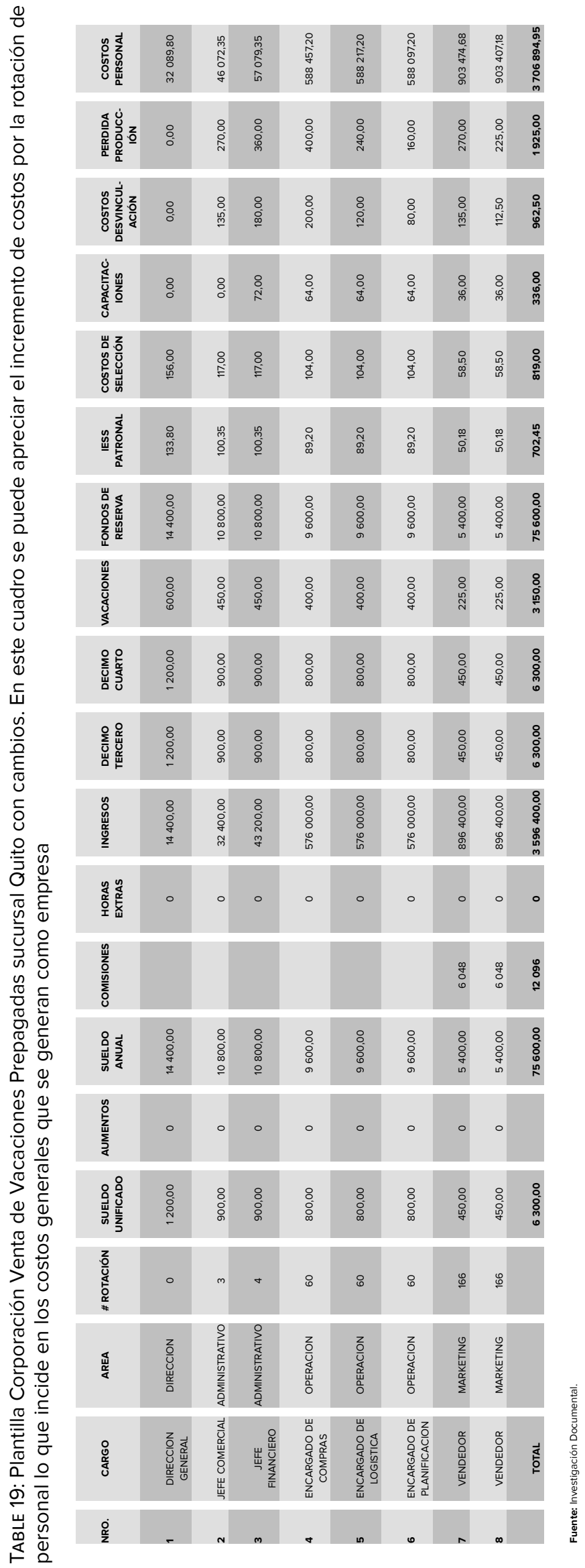


evaluación del desempeño. Quito, Pichincha, Ecuador: Universidad Andina Simón Bolivar.

[3] Arias Galicia, F. (2001). El compromiso personal hacia la organnización y la intención de permanencia: algunos factores para su incremento. Contaduría y Administración, 200.

[4] Becker, B. E., Huselid, M. A., \& Ulrich, D. (2002). El cuadro de mando de rrhh. Barcelona: Gestion 2000.

[5] Becker, B., Huselid, M., \& Dave, U. (2000). El cuadro de mando de rrhh. Barcelona: Gestion.

[6] Bernal, C. (2006). Metodologia de la Investigacion para la administracion, economia, humanidades y ciencias sociales. Mexico: Pearson.

[7] Betanzos Días, N., \& Paz rodríguez, F. (2007). Análisis psicométrico del compromiso organizacional como variable actitudinal. Anales de Psicología, 207-2015.

[8] Bohlander George, S. S. (2007). Administración de Recursos Humanos (14 ed. ed.). México D.F, México, México: Cengage Learning.

[9] Brian E, B., Mark A, H., \& Ulrich, D. (2002). El Cuadro de Mando de Recursos Humanos. Bareloona: Gestion 2000, S.A.

[10] Brizius, J., \& Campbell, M. (1991). Getting results: A Guide for Government Accountability. Washington: Council of Governors.

[11] C. McKinsey Quarterly. (Agosto de 2010). McKinsey\&Company. Recuperado el Noviembre de 2009, de Retaining key employees in times of change: http://www.mckinsey.com/insights/organization/ retaining_key_employees_in_times_of_change

[12] Carranza, F., Maldonado, S., \& Guillen, A. (2011). EL COMPROMISO ORGANIZACIONAL DEL PERSONAL ADMINISTRATIVO EN UNA UNIVERSIDAD PÚBLICASO. REVISTA INTERNACIONAL DE ADMINISTRACIÓN Y FINANZAS, 11.

[13] Carvajal, L. (18 de Enero de 2013). Lizardo Carvajal. Obtenido de Lizardo Carvajal: http://www.lizardo-carvajal.com/el-metodo-deductivo-de-investigacion/

[14] Castillo, J. (2006). Administración de Personal: Un Enfoque hacia la Calidad. Bogota: Ecoe Ediciones.

[15] Cervera, J. (2002). La Transición a las nuevas ISO 9000-2000 Y SU IMPLANTACION. España: Diaz de Santos.

[16] Chiang V, M., Núñez P, A., Martín, M., \& Salazar B, M. (Julio de 2010). Compromiso del Trabajador hacia su organización y la relación con el Clima Organziacional: Un análisis de género y edad. Panorama Socioeconómico, 92-103. 
[17] Chiang Vega, M., Martin Rodrigo, M., \& Nuñez Partido, A. (2010). Relaciones entre el Clima Organizacional y la Satisfaccion Laboral. Madrid: Universidad Pontificia Comillas.

[18] Chiavenato, I. (2001). Administración de Recursos Humanos (5ta. ed.). Santa Fé de Bogotá, Colombia: McGraw-Hill Interamericana S.A.

[19] Chiavenato, I. (2009). Comportamiento Organizacional. Mexico: McGraw Hill.

[20] Garcia Cruz, J., \& Real Fernández, J. (26 de Julio de 2013). Confianza como consecuencia del compromiso percibido: Implicaciones sobre el aprendizaje y la innovación. Cuadernos de Gestión Vol. 13, 95-124.

[21] García Jesús, S. R. (2004). Fundamentos de Dirección y Gestión de Recursos Humanos. España: Thomson.

[22] Gibbs, E. L. (2011). Economia de los recursos humanos en la práctica. España: Antoni Bosch.

[23] Hellriegel, D., Jackson, S. E., \& Slocum, J. S. (2002). Administración Un Enfoque Basado en Competencias (9na. ed.). (P. De la Garza, \& A. Gómez Ruiz, Edits.) Bogotá, Colombia: Thomson Editores.

[24] Hernandez Sampieri, R. (2007). Fundamentos de la metodología de la investigación. México: MCGRAW-HILL.

[25] Hernandez Sampieri, R., Fernandez Collado, C., \& Baptista Lucio, P. (2006). Metodologia de la Investigación. Mexico D.F.: McGraw-Hill / Interamericana Editores S.A. de C.V.

[26] Hernández Sampieri, R., Fernández Collado, C., \& Baptista Lucio, P. (2006). Metodología de la Investigación. México D.F.: McGraw-Hill / Interamericana Editores S.A. de C.V.

[27] Hernandez, R. (2010). Metodología de la Investigación. Mexico DF: McGraw Hill.

[28] Hunt, J. (1993). La Dirección de Personal en la Empresa. Madrid: McGrawHill/Interamericana de España, S.A.

[29] Idalberto, C. (2000). Administración de Recursos Humanos (5ta. ed.). Santa Fé de Bogotá, Colombia: McGraw-Hill Interamericana S.A.

[30] Idalberto, C. (2000). Administración de Recursos Humanos. Colombia: Mc Graw Hill.

[31] Sagi, L., \& Vela, G. (2011). Gestión por competencias. Madrid: Esic.

[32] Sampieri Roberto Hernández, F. C. (2010). Metodología de la Investigación (5ta. ed. ed.). México D.F., México, México: McGraw-Hill. 
[33] Viteri, D(2016) Impacto Financiero De La Rotación De Personal En Las Áreas De Mercadeo Y Operaciones De Una Empresa Multinacional De Venta De Vacaciones Pre Pagadas En El Ecuador, Tesis Repositorio UTE.

[34] W. Moulton. (Febrero de 2015). KompeDisc. Psigma Corp.

[35] Werther William B., D. J. (2003). Administración de Personal y Recursos Humanos (5ta. ed. ed.). México, México, México: Mc Graw Hill.

[36] Werther, D. y. (1995). Administración de personal y recursos humanos. McGraw-Hill Interamericana.

[37] Williams, M., Unrau, Y., \& Grinnell, Y. (2005). The qualitative research approach. Nueva York: Oxford University Press. 